\title{
Wie können Sie (Privat-)Patienten an Ihre Praxis binden?
}

\author{
Für den wirtschaftlichen Erfolg einer Facharztpraxis sind eine \\ florierende Privatpraxis und die Erschließung von Wachstums- \\ potenzialen außerhalb der GKV-Abrechnung notwendig geworden. \\ In einer Studie zur Patientenzufriedenheit wurden Kriterien \\ herausgearbeitet, die für Privatpatienten bei der Praxiswahl bzw. \\ dem Wechsel einer Arztpraxis maßgebend sind.
}

— In einer „Zufriedenheitsstudie“ hat das Institut für Management- und Wirtschaftsforschung in Kooperation mit dem Unternehmen SGS-TÜV mehr als 1000 Privatpatienten gefragt, welche Aspekte für sie bei der Auswahl einer neuen Praxis und beim Arztwechsel am wichtigsten sind. Die Ergebnisse zeigen, dass der Unternehmenserfolg des Arztes selbstverständlich von seinen medizinischen Leistungen abhängt. Für die Patienten sind aber auch Kriterien maßgebend, die der Facharzt bei seinem unmittelbaren Kontakt mit den Patienten nicht beeinflussen kann.

Zugespitzt ausgedrückt: Die besten medizinischen Leistungen des Arztes nutzen wenig, wenn das Praxisteam bei der Betreuung des Patienten - etwa bei Terminvereinbarungen - gravierende Fehler macht. Bei den fünf Topgründen für die Praxiswahl geht es den Patienten eher um „weiche Faktoren“" wie die Bewertung des Praxismanagements (siehe Tabelle 1).

\section{Häufige Praxiswechsel}

Die Bindung der Privatpatienten an die Praxis ist eine anspruchsvolle Aufgabe. Auf der einen Seite zeigt die Studie, dass Vertrauen (38\%) bzw. eher großes Vertrauen (48\%) der Arztpraxis entgegenbringen, die sie am häufigsten aufsuchen. Auf der anderen Seite muss der Facharzt damit rechnen, dass die Privatpatienten eine anspruchsvolle Klientel darstellen, die ihren Sonderstatus im Gesundheitswesen kennt und bereit ist, bei Unzufriedenheit mit dem Arzt oder der Praxis sehr rasch einen anderen Arzt aufzusuchen.

Rund $45 \%$ der Privatpatienten haben in den letzten fünf Jahren wegen Unzufriedenheit mindestens einmal den Arzt oder die Praxis gewechselt. Die Wechselbereitschaft ist bei jüngeren (vor allem auch weiblichen) Patienten besonders ausgeprägt. Gut ein Fünftel der Privatpatienten gibt in der Untersuchung an, in den letzten $86 \%$ der Privatpatienten ein sehr großes

\begin{tabular}{|l|l|}
\hline $\begin{array}{l}\text { Aspekte für die Auswahl einer neuen Arztpraxis } \\
\text { Freundlichkeit/Verständnis des Personals und des Arztes }\end{array}$ & Tabelle 1 \\
\hline Qualifiziertes Praxisteam & $53 \%$ \\
\hline Angemessene Betreuungsdauer durch den Arzt & $50 \%$ \\
\hline Einhaltung von Terminen/kurze Wartezeiten vor der Behandlung & $44 \%$ \\
\hline Verständliche Information über die Notwendigkeit einer Behandlung & $44 \%$ \\
\hline Verständliche Information über die einzelnen Behandlungsschritte & $42 \%$ \\
\hline Zeitgerechte Terminvergabe in Abhängigkeit von meinem Anliegen & $42 \%$ \\
\hline Kundenfreundliche Praxiszeiten (z. B. auch abends und am Wochenende) & $40 \%$ \\
\hline
\end{tabular}

fünf Jahren dreimal oder öfter die Arztoder Facharztpraxis gewechselt zu haben.

\section{Hauptgründe für den Praxiswechsel}

Der Arzt muss wissen, dass die Leistungsfähigkeit seines Unternehmens von den Patienten sehr aufmerksam beobachtet wird. Ein hoher Anteil der Patienten hat schon mehrere Arztpraxen kennengelernt und dabei Vergleichsmaßstäbe entwickelt. $\mathrm{Zu}$ einem Arztwechsel entschließen sich Privatpatienten, wenn sie bei der Behandlung Unfreundlichkeit und mangelndes Verständnis des Arztes oder seines Praxisteams empfinden (67\%). Auch Mängel bei der Qualifikation des Praxisteams sind für viele Patienten ein Grund für den Arztwechsel (55\%), ebenso wie Probleme in der zeitgerechten Terminvergabe (52\%) bzw. lange Wartezeiten (50\%) - Fehler, die sich vermeiden lassen.

\section{So gelingt die Bestandssicherung}

Die beste Marketingmaßnahme zur Bestandssicherung und um neue Patienten zu gewinnen ist die Zufriedenheit der eigenen Klientel. Für das Praxisteam kommt es darauf an, die Privatpatienten optimal zu betreuen, ohne dabei Konflikte mit den Kassenpatienten zu riskieren.

Bei der Praxiswahl dominiert zunächst die örtliche Nähe der Praxis. Die Patienten sind aber heutzutage durchaus bereit, auch weite Wege in Kauf zu nehmen, wenn es einem Arzt mit seinem Praxisteam gelungen ist, einen guten Ruf in der Region zu erwerben.

\section{Fazit für die Praxis}

Nach den Ergebnissen der Studie muss sich der Facharzt um ein optimales Management bei seiner Privatpraxis kümmern. Freundlichkeit der Mitarbeiter gegenüber den Patienten, ein qualifiziertes Praxisteam und Entgegenkommen bei Terminvereinbarungen und Wartezeiten sind zentrale Anforderungen an eine erfolgreiche Unternehmensführung. red 